total of 11,295 men are not at present available, as the returns from the different regiments did not in every case give the result of the illness. From the cases in which the issue of the illness was stated the mortality among the inoculated was calculated at two per 1000 and among the nninoculated at 3.4 per 1000 . The case mortality was at the rate of 185.2 per 1000 among the inoculated and 1033 among the uninoculated. These figures are undoubtedly in farour of the inoculated and might have been more strongly so had it been possible to have classed the men as " successfally inoculated" and "others," instead of as simply "inoculated" and "uninoculated." For with the methods in use at present the inoculation is unsuccessful in a certain proportion of the cases, and those who after inoculation do not give a good agglutinative reaction with their serum cannot for statistical purposes be fairly classed amongst the "inoculated."

Of the results of the inoculations which have been carried out in the case of the troops in South Africa it is too early to form any opinion. Mr. Osborn, ${ }^{5}$ in commenting on the large number of cases of typhoid fever which have occurred among the troops, stated that at Modder River the disease occurred at the rate of 6 per 1000 among those who had been inoculated and at the rate of 9 per 1000 among those who had not. No stress can be laid on these last figures one way or another, but extremely valuable data will be available after the war when the complete returns are forthcoming. And in connexion with these statistics it is especially to be hoped that a thorough effort will be made to ascertain by means of the serum test what proportion of the soldiers who underwent the operation on the way out had really reacted to the inoculation. It has been said that the active immunity which follows the injection of a dead culture in animals takes some time to develop and that after the inoculation in man a definite interval elapses before the agglutinative property is manifested in the serum. It is not, therefore, to be expected that any protective effect will follow the operation immediately. I do not think, for instance, that protection would be afforded against an infection within about a week after the inoculation. In speaking of this I would particularly refer to Case 1 on the temperature chart. In this case there had been exposure to the risk of infection under rather exceptional circumstances both before and after the inoculation, the patient being one of four who contracted the disease by direct personal infection from one individual. As far as one could judge the patient must have been inoculated some five or six days after infection had occurred, and definite symptoms of the disease appeared on the eleventh day after the inoculation. The patient passed through an ordinary mild attack of the disease. It will be noticed that the temperature reaction after inoculation in Case 1 on the temperature chart differs very distinctly from the type occurring in Cases 2, 3, and 4, which were inoculated at the same time and from the same sample of serum.

At present we do not know exactly what length of time any protection afforded by the inoculation may be expected to last. In a certain proportion of cases, if we accept the persistence of agglutinative power as an indication of the persistence of immunity, the protective effect lasts for at least two years. By the kindness of Dr. Arthur Jackson, assistant medical officer to the Kent County Asylum, I had the opportunity in October last year of examining the blood of nine of those who had been inoculated jast 24 months previously. The results obtained on testing the agglutinative power of the serum in these nine cases are shown in the following table which has already been published elsewhere. ${ }^{6}$ of the nine cases four had previously been tested by Dr. Tew ${ }^{\tau}$ and two of them (Case 4 and Case 5) had given only doubtful reactions shortly after inoculation; the serum from the last five cases had not been tested previously.

From what I have said it will have been gathered that the immediate after-effects of an anti-typhoid inoculation may be distinctly unpleasant, and it is not suggested that these inoculations should be employed generally, but only for those who may be specially liable to the risk of in. fection. And among those to whom preventive inoculation is likely to afford most benefit are nurses in

S. Osborn: Hospital Arrangements in the South African War THE LAYCET, April 21st, 1900, p. 1158.

Foulerton: On the Serum Treatment of Enteric Fever and Bacterial Prophylactic Inoculation, Middlesex Hospital Journal, vol. iii. To, 4,1899 . Loe. cit. hospitals into which cases of typhoid fever are admitted, and soldiers who are serving in India and other places where this disease is most prevalent. And it need scarcely be added that if inoculation is advisable for

\begin{tabular}{|c|c|c|c|c|c|}
\hline \multirow{2}{*}{ Case. } & \multirow{2}{*}{ Sex. } & \multicolumn{4}{|c|}{ Dilution of serum used in testing for agglutination. } \\
\hline & & 1 in 10. & 1 in 50 . & 1 in 100 . & 1 in 200 . \\
\hline 1 & M. & - & - & - & $\begin{array}{l}\text { Instantaneous } \\
\text { reaction. }\end{array}$ \\
\hline 2 . & F. & - & - & - & $\begin{array}{l}\text { Instantaneous } \\
\text { reaction. }\end{array}$ \\
\hline 3 & F. & - & - & $\begin{array}{l}\text { Instantaneous } \\
\text { reaction. }\end{array}$ & Not tested. \\
\hline 4 & F. & - & $\begin{array}{l}\text { Reaction } \\
\text { within } 20 \\
\text { minutes. }\end{array}$ & No reaction. & - \\
\hline 5 & M. & No reaction. & - & - & \\
\hline 6 & F. & , & - & - & - \\
\hline 7 & F. & ,, & - & - & - \\
\hline 8 & F. & ", & - & - & - \\
\hline 9 & M. & ", & - & - & - \\
\hline
\end{tabular}

a nurse or a soldier the younger the individual may be the more strongly is inoculation to be advised. And it must not be forgotten that these two classes which I have mentioned include those who are not safeguarded by ordinary sanitary measures. In this country typhoid fever is essentially a water-borne disease and the majority of us can rely for protection against infection upon the work of our medical officers of health. But the nurse is brought into intimate contact with patients who are suffering from the disease, and those who have had long experience of hospital work will well know that there is in some cases such a thing as personal infectivity in typhoid fever, and that quite apart from careless or unskilful nursing there is a real risk in personal contact. Soldiers living in camp in hot dry climates where typhoid fever is an air-borne as well as a water-borne disease also require special protection. In such a climate it would seem under the prevailing conditions all but impossible to prevent the dissemination to some extent of particles of organic matter from latrines by the wind.

Finally, it may be pointed out that whilst the immediate effects of inoculation may be, and often are, decidedly unpleasant they are merely temporary, lasting only for a few hours, and up to the present time have never had any more serious result than the short period of discomfort. It need scarcely be added that if the most ordinary care has been observed in the preparation of the propbylactic fluid there is not the very remotest chance, either in these antityphoid inoculations or in the similar procedure against cholera and bubonic plague, of conveying the disease itself. In every case in which it is desired that there should be a prospect of protection lasting for more than two years I think that it is desirable to ensure a good tissue reaction by repeating the anti-typhoid inoculation. The second dose may be given a week after the first, and the agglutinative reaction of the blood tested after a further interval of a few days.

Sutton, Surrey.

\section{A CONTRIBUTION TO THE STATISTICS OF RHEUMATIC FEVER AND ITS COMPLICATIONS.}

BY WILLIAM CECIL BOSANQUET, M.A., M.D. OXON., M.R.C.P. LOND.,

FORMERLY FHLLOW OF NEW COLLEGE, OXFORD; ASSISTANT PHYSICIA TO THE VICTORIA HOSPITAL FOR CHILDREN ; MEDICAL RHGISTRAR, CHARING-OROSS HOSPITAT.

THE total number of cases of rheumatic fever admitted into Charing-cross Hospital in the course of eight years (1890-97 inclusive) was 450.

Sex and age.-Of the whole number 237 were males and 213 were females, from which it might appear that both sexes are equally liable to the disease. Since, however, there are nearly twice as many male as female beds in the hospital it is probable that these numbers really show a greater liability 
of the latter sex to suffer from rheumatism. The following table shows the age-incidence of the cases as admitted to hospital :-

\begin{tabular}{cccc|c|c|c|c|c|c}
\hline & & & $\begin{array}{c}1 \text { to } 10 \\
\text { years. }\end{array}$ & $\begin{array}{c}11 \text { to } 20 \\
\text { years. }\end{array}$ & $\begin{array}{c}21 \text { to } 30 \\
\text { years. }\end{array}$ & $\begin{array}{c}31 \text { to } 40 \\
\text { years. }\end{array}$ & $\begin{array}{c}41 \text { to } 50 \\
\text { years. }\end{array}$ & $\begin{array}{c}51 \text { to } 60 \\
\text { years. }\end{array}$ \\
\hline Males & $\ldots .$. & $\ldots$ & $\ldots$ & 11 & 78 & 79 & 43 & 21 & 5 \\
\hline Females ... &... & $\ldots$ & 8 & 86 & 82 & 26 & 9 & 2 \\
\hline
\end{tabular}

By far the greater number of cases thus occur between the ages of 10 and 30 years, no less than 157 males and 168 females being included in this period, or over 72 per cent. of the whole number. It is noticeable that within these limits the female cases, in spite of the smaller number of available beds, slightly outnumber those of the other sex. It may be right to point out that the incidence of the disease on children under 11 years may not be fully represented for several reasons. In the first place, the number of children's medical beds in the hospital is only nine and the population from which they are filled is possibly more limited than in the case of adults, owing to the preference of parents for special children's hospitals. Further, it cannot be doubted that the disedse often passes unrecognised in children owing to the ill-marked joint-symptoms to which it gives rise at this time of life and the fact that this disease is not popularly associated with childhood. Several instances have come under my own observation in which an illness occurring in a child which was almost undoubtedly rheumatic fever, accompanied by actual swelling of the joints and subsequent valvular disease of the heart, was called "influenza" at the time. In another instance a similar attack was called " malaria"; and it is practically certain that many mild cases of rheumatism which may yet leave serious sequelæ are either entirely overlooked or are treated as mere "feverish attacks," " biliousness," or other creations of household medicine. I might even be suggested that some of the success of salicylate of sodium in the treatment of influenza and some of the cases of cardiac mischief attributed to this protean plague are in reality to be attributed to errors of diagnosis in cases of genuine rheumatism.

Taking first attacks only, including those patients who stated that they had never had a previous attack, and accepting the accounts given by others who were able to fix the date of their first experience of the disease (a total of 304 cases), it appears that no less than 156 (50 per cent.) of these first attacks fell between the ages of 11 and 20 years (females 79 , males 77). Under 11 years, 17 cases occurred in male and 21 in female children. From 21 to 30 years there were 42 first attacks in males and 39 in females. The earliest recorded instance among the number was a boy who was reported to have had rheumatism at the age of three weeks. Other early cases were those of a girl aged two years, a boy aged three years (actually observed in hospital), and a girl and boy aged four years respectively. The latest occurrences said to represent first attacks were at 53 and 57 years, both in men. Only one first attack oscurred in a woman over 40 years of age.

Heredity.-No very definite reliance can be placed on the family history of these patients, the accounts given of the health of parents and relatives being as a rule untrustworthy, especially with regard to a common and not fatal illness such as rheumatism. As a matter of fact in 99 cases, or approximately 22 per cent., the patients gave a history of rheumatism in one or more members of their families.

Ocoupation.-With regard to the influence of employment and mode of life only one fact appears at all prominently, namely, the great number of domestic servants who suffer from rheumatism. Out of the present 450 cases no less than 105 ( 23 per cent.) occurred in this class, 85 being female servants and 20 males. If we add to this number 19 cases of waiters, 10 of barmaids, four of male cooks, and two of office boys, we have a total of 140 cases out of 450 , or 31 per cent., belonging to this class of society, while of females alone nearly 40 per cent. were in service. ${ }^{1}$ No special liability to rheumatism in those exposed to vicissitudes of weather was evident in this series of cases. The reason for the special incidence on women between 10 and 30 years of age can

1 The ratio of service to other occupations among 415 female patients admitted during 1898 whose occupations were stated was 26 per cent. which may be taken as the ordinary proportion among patients in this hospital. only be surmised; but the prevalence of anæmia at this period is a very marked coincidence, while both of these diseases seem to fall specially on the servant-class of society. It may be suggested that the existence of anæmia acts as a predisposing cause of acute rheumatism. In this connexion it is interesting to note that in one case, that of a woman, 42 years of age, several attacks had occurred, each in connexion with pregnancy, the period of lactation being always accompanied by rheumatic symptoms. Here, too, perbaps, the anæmia brought about by this drain on the system acted as the predisposing cause. This might well be the case if rheumatic fever is due to the action of some micro-organism, as seems highly probable, although there is little evidence of any transmission of the disease from one individual to another.

Season.-As far as can be gathered from the limited number of cases here recorded rheumatic fever has two periods of maximum incidence in the year corresponding to the months of May and November. That a much larger series is necessary before it is possible to construct a true curve of incidence for the year seems shown by the sudden drop that occurs in the present record between May and June, these months having 47 and 29 cases respectively, or practically the highest and lowest numbers thronghout the 12 months (November, the actual maximum, shows 48). It is worthy of remark that while the admissions to this hospital of cases of enteric fever and of acute pneumonia correspond very closely with the accepted periods of maximum frequency of these diseases the cases of rheumatism do not correspond so closely. Probably the seasonal curve of rheumatism is less well defined than that of either of the two other maladies. It is to be remarked that the two months showing the highest averages of cases are months associated, as a rule, with chilly and unsettled weather, when there occur constant changes of temperature.

Onset.- In the vast majority of cases the first thing complained of by the sufferers from rheumatism was pain in one or more joints, which generally began gradually and only caused them to take to bed after one or more days. In a certain number of cases sore-throat was the first thing noticed, apparently in about 5 per cent. Vomiting occurred early in about 3 per cent. of the cases, and shivering or actual rigor in about the same proportion. In approximately 5 per cent. headache was a prominent symptom at the beginning of the illness. Its occurrence later as a result of treatment with salicylates is too well known to need mention and cannot be considered a symptom of the disease.

Another mode of onset, but by no means a common one was with symptoms of acute pneumonia which masked the pains in the joints, while in one case (that of a girl, aged 14 years) the onset was gradual without joint-pains, but with headache and general malaise, suggesting enteric fever. In another instance enlargement of the spleen was noticed which, if it had occurred along with the former insidions onset, would have caused a misleading resemblance to enteric fever before the occurrence of the typical articular affection.

Duration.-With regard to the duration of the disease it is difficult to say anything definite. When once admitted to hospital the temperature in most cases fell within two or three days, and the pains vanished simultaneously under the influence of salicylate-treatment. On the other hand, patients gave histories of their "rheumatics" having lasted for months before they came for treatment. It is impossible to determine whether these cases are to be considered instances of one long attack or, as seems more probable, as constituting a succession of relapses. Accepting the statements of the patients and calculating from their account of the beginning of their symptoms, the larger number, or nearly two-thirds of the whole, were free from pain and fever within two weeks; but it is impossible to say how long they would have been ill if, on the one hand, they had not come for treatment, or, on the other hand, if they had been treated sooner. Occasionally, even in hospital, cases proved very refractory: in one instance in the present series the patient (a male, 20 years of age) was seven weeks ill in hospital; others were as long as 33 and 39 days, with continued fever and pain in spite of the usual treatment. In two patients, both females, 26 and 36 years old respectively, the disease ultimately settled in one joint and became chronic, the patients leaving the hospital with knees still stiff and painful.

Toints affected.-It is recognised as a feature of the disease that it affects many joints in succession, "flying from one to 
another," but on surveying a number of cases it shows a manifest predilection for certain seats. Thus, of about 430 cases in which the affected joints were recorded the kneejoint was affected in 297 instances (69 per cent.), the ankle in 202 (47 per cent.), and the shoulder in 115 (26 per cent.). The elbow, the wrist, and the small joints of the hand and the foot were affected with nearly equal frequency (from 80 to 100 cases); the hip came next with 54 cases and the spine with 37 cases. The neck was affected 11 times, the sterno-clavicular joint eight times, and the temporo-maxillary articulation twice. In 24 cases all the joints were said to be painful, but this vague statement probably only signified the general discomfort and misery of the sufferers. It thus appears that the knee and ankle are nearly twice as oiten affected as any other joints. The hip-joint, a very frequent seat of rheumatoid arthritis, is not often attacked in rheumatic fever. In a few instances tranmatism of a joint (strain, \&c.) seems to have determined the seat of onset of the malady.

Complications. - The most frequent complication of acute rheumatism-if it is to be called a complication and not, as seems more probable, a distinct feature of the disease-is undoubtedly endocarditis, and in the present series of cases it was found in 67 males ( 28 per cent.) and 70 females ( 33 per cent.) either as recent endocarditis or old valvular disease. In the former sex the mitral valve was affected alone 51 times, the aortic alone in four cases, and both together in 12 instances. In the females the aortic valve suffered alone on two occasions and combined mischief was only met with once, the rest being pure mitral cases. Ulcerative endocarditis occurred in three patients.

Pericarditis was met with in 17 males and 11 females. Of these three died, but in only one of the fatal cases, that of a girl, aged ifive years, was pericarditis the sole complication, the other two patients having pneumonia, associated in one case with hyperpyrexia. If we add to the above 28 cases of pericarditis 32 others occurring in the hospital in the same period we find that out of a total of 60 cases of this affection 46 (nearly 77 per cent.) occurred in the course of acute rheumatism or were considered "rheumatic." The total mortality of the 60 cases was 19 or nearly 32 per cent., but in four of these the disease was associated with pneumonia, in two with phthisis, and in two with granular kidneys and cirrhosis of the liver respectively. The deaths, therefore, in rheumatic cases, apart from other complications, were nine out of 46 patients, or nearly 20 per cent. On the other hand of 10 cases associated with pneumonia six patients died, or 60 per cent. Two suppurative cases occurred in rheumatic subjects, included in the above numbers, and were both fatal; but they were not associated with symptoms of rheumatic fever at the time of their occurrence. If they were subtracted from the above numbers the mortality of sero-fibrinous pericarditis complicating rheumatic fever would appear to be only about 15 per cent.

The frequency of the occurrence of relapse in cases of rheumatism is difficult to estimate, owing to the fact noticed above that many of the instances of long duration of the illness before admission to hospital are probably due to a series of relapses. Actual instances could be made sure of in 42 cases, or roughly 10 per cent., but the figures are not of much value.

Pneumonia occurred in 14 cases of the present series, equally divided between the two sexes. Of these two, accompanied by pericarditis, were fatal. The total number of cases of pneumonia occurring in the hospital in the period recorded was 300 and in 24 of these- 8 per cent-there was more or less reason to associate the disease with rheumatism. It is an interesting speculation to what extent the two diseases are connected, and it is permissible perhaps to surmise that in cases of pneumonia, especially those which run a rather atypical course and end by lysis, closer search would reveal a rheumatic predisposition more often than is at present suspected. In only two of the above 24 cases did the disease end by crisis. The value of salicylate of sodium in acnte pneumonia is also of interest in this connexion.

Hyperpyrexia occurred in two cases only, in one of which it was associated with pneumonia. The number seems small, and it is possible that early treatment with salicylates prevents a more frequent occurrence of this fatal complication. Plenrisy was found 14 times and bronchitis in 22 cases; in one patient the latter malady seems to have been responsible for a fatal issue.

Chorea occurred in two males and 11 females, giving an average of nearly 3 per cent. The oldest patients were two girls, 19 years of age. Delirium was noticed in nine cases, chiefly males, several times associated with alcoholic habits, but not always so. In two women there was troublescme neuralgia of the face. Severe abdominal pain was complained of in 13 cases without obvious cause, and diarrhœea occurred in two instances. Four cases presented symptoms of nephritis, two being of the chronic interstitial variety, one a subacute tubal nephritis and one an acute attack; this last occurred in the hospital without any cause that could be discovered other than the rheumatic fever. A purpuric eruption occurred in two cases and hæmaturia in three others, probably due in one case to embolism and in another very possibly to papilloma of the bladder. Epistaxis was twice observed. In one case blood was passed per rectum without obvious cause other than the general disease. Phlebitis occurred once and arterial thrombosis (endarteritis?) affecting the temporal and occipital arteries also in one instance. Of skin-affections, erythema nodosum ${ }^{2}$ was observed in nine instances (2 per cent.), all females, and other forms of erythema multiforme in four others (three being women); in two cases it was possibly due to the effect of salicylic treatment. Subcutaneous nodules occurred in five cases, the oldest patient being a woman 26 years of age. In one instance they were the seat of considerable pain. Accidentally associated diseases were gout in six cases (showing that the two diseases are not incompatible), phthisis, Graves's disease, epilepsy, tabes dorsalis, cirrhosis of the liver, and ague.

Death.-In no case of the present series did death occur from an uncomplicated attack of rheumatic fever. Seven cases proved fatal altogether (about 15 per cent.), six patients being males and one a female. Two of these cases were due to pneumonia accompanied by pericarditis, one to pericarditis alone: hyperpyrexia accounted for one more. Two cases proved fatal owing to ulcerative endocarditis and one to bronchitis.

Treatment.-With regard to this little need be said. Rest in bed, fluid diet, and salicylate of sodium constituted the routine treatment in all cases. It was often possible to trace a close connexion between the occurrence of a relapse and an unduly early return to fuller diet or a premature omission of the specific drug. In obstinate cases alkalies were tried but did not seem to have much effect. Iodide of potassium, however, occasionally seemed serviceable, aided by blistering of the affected joints in very chronic cases. A recent patient not included in the above series, appeared to derive benefit from treatment with hot air, a method which is certainly worthy of trial in many cases of chronic joint-disease.

Harley-street, w.

\section{ON OPERATIONS FOR CANCER IN THE} AGED.

By E. HARDING FREELAND, F.R.C.S. ENG., SURGEON TO THE ST. GEORGI'S AND ST. JAMHS'S DISPENSARY, LONDON.

IN The Lancet of May 5th, 1900 (p. 1276), there appeared a paper by Mr. T. Frederick Gardner entitled "Three Cases of Surgical Interest." The first of the series-a scirrhous cancer of the breast-raises the question of the advisability of operating for cancer in extreme old age. This case has for me a peculiar interest, as a similar case occurred in my practice about two years ago which, taken in conjunction with Mr. Gardner's case, may be worth recording and prove interesting to others.

A spinster, aged 82 years, thin, wiry, and intelligent, somewhat feeble, but for her years in fairly good bealth, consulted me on March 18th, 1898, for a painful tumour in the left breast, which, she said, she had noticed about a fortnight previously. She told me that she had been "out of sorts" for several months and she thought that she had been losing flesh. On examination I immediately found a nodular swelling of stony hardness of about the size and shape of a plover's egg in the left breast, situated about an inch below, and external to, the nipple. The skin over the tumour was freely moveable except at one point

3 The total number of cases of erythema nodosum admitted in the eight years was 20 , of which only three were males, aged respectivel 12,14 , and 17 years. The oldest female patient was aged 31 years, th youngest seven years.
glaadular enlargement. 